\section{On the Jobs}

When it comes to desirable features in a career-oriented site, the primary consideration that matters is listings. With over 11,000 positions at over 1600 institutions of higher learning on its site, HigherEdJobs.com makes a strong case for academicians to take a serious look at it. Among the sciences, over 1000 different faculty positions are available, including over 250 in the biological sciences. HigherEdJobs provides free registration and resume posting for job seekers. And if you don't feel like checking the listings every day, e-mail notifications are available. An extensive list of institutional profiles rounds out the offerings.

www.higheredjobs.com

\section{Comparative Exonomy}

If protein evolution is facilitated by shuffling of exon sequences, it follows that a considerable amount of information can be obtained by studying intron/exon boundaries of protein coding regions. The Structural Exon Database (SEDB) paired with the companion program Friend has its finger on the pulse of this topic. Hosted at Northeastern University, SEDB/Friend helps users identify related sequences, align them, and then align the related structures. Searches in SEDB can be performed by Protein ID, Genbank Accession, Structure, Sequence, or BLASTing against SEDB. In Friend, structures, alignments, and phylogenetic trees are viewed and hydrogen bond predictions are made.

\section{glinka.bio.neu.edu/SEDB/SEDB.html}

\section{A Peptide Runs Through It}

Its plot lines can be pretty slim and it may not win any Academy Awards. Neither will it be used to create a box office smash, but the Protein Movie Generator (version 2.0) is a hit among protein scientists for the tools it provides to animate the movement of complex polypeptide structures in 3-D space. Users start very simply with a PDB identification tag and then build a movie by specifying parameters. A ray tracing program called megapov provides the horsepower. Numerous options are provided for displaying peptide backbones, sidechains,

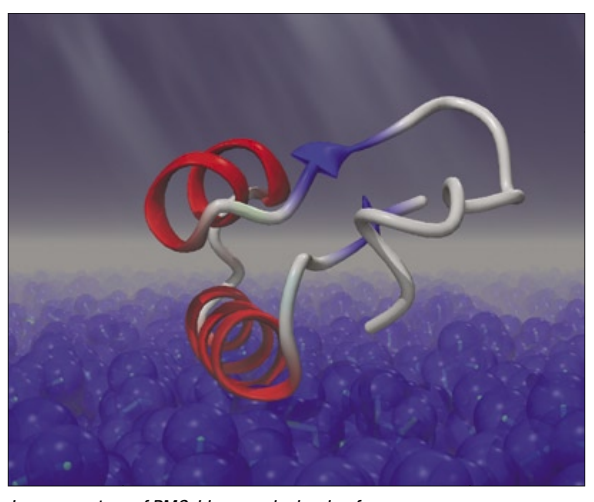

Image courtesy of PMG, bioserv.rpbs.jussieu.fr.

surfaces, and other features. Opacities can be varied, as well as color, backgrounds, and number of frames. If sitting in the director's seat for a peptide-based motion picture is your dream, the Protein Movie Generator is for you. Bring on the popcorn.

\section{bioserv.rpbs.jussieu.fr/ autin/ help/PMGtuto.html}

\section{iHop, Skip, and Jump}

You search through a sequence database and locate a gene of interest, but then a forest of links appears that leads in dozens of different directions. Can you say information overload? Hyperlinks are great for identifying related data, but navigating through them, tracing your steps, and efficiently locating what you're after can be a chore. Recognizing this, the designers of iHOP took a radically different approach to organizing protein data. First, protein entries in the database are described in short sentences, with links inside them color-coded as to what they lead to.
Tan ones, for example, link to multivariable searches of PubMed and blue ones point to other genes. A site that makes it hard to get lost, iHOP provides improved tools for accessing information.

\section{www.ihop-net.org/UniPub/iHOP}

\section{What It's All About-Algae}

An amazingly diverse set of organisms, the algae are hard to categorize and, in fact, are not derived from a single evolutionary line. Fortunately that hasn't impeded Webmasters. A group that contains over 120,000 species, the algae are photosynthetic multicellular and unicellular organisms commonly divided by color and are distinct from land plants in that they lack leaves, roots, and other organs. They have been investigated for being protein sources, as well as a new source of biofuel. Algaebase, a database with information spanning the terrestrial, marine, and freshwater forms of these organisms, provides visitors with reference, taxonomic, and other information. It's the place to shop when you're in the market for algae information.

\section{www.algaebase.org}

-Kevin Ahern - Please send web site recommendations to ahernk@orst.edu 